\title{
Progression of coronary artery disease in a HIV-infected patient previously treated for ascending aorta aneurysm
}

\author{
Radosław Zwoliński ${ }^{1}$, Juliusz Kamerys ${ }^{2}$, Elżbieta Jabłonowska ${ }^{3}$, Anna Marcinkiewicz ${ }^{1}$, Ryszard Jaszewski ${ }^{1}$, \\ Radosław Kręcki ${ }^{4}$, Bogdan Jegier ${ }^{1}$ \\ ${ }^{1}$ Department of Cardiac Surgery, Central Clinical Hospital, Medical University of Lodz, Lodz, Poland \\ 2Department of Infectious, Tropical and Parasitic Diseases, Biegański Hospital, Lodz, Poland \\ ${ }^{3}$ Department of Infectious Diseases and Hepatology, Medical University of Lodz, Lodz, Poland \\ ${ }^{4}$ Department of Cardiology, Medical University of Lodz, Lodz, Poland
}

Kardiochirurgia i Torakochirurgia Polska 2017; 14 (3): 211-213

The pathophysiology of increased cardiovascular risk in HIV infection is complex and multifactorial but chronic inflammation and immune activation seem to play a crucial role. Direct effects of HIV, leakage of bacteria from the gut, damage of lymphoid tissues as well as co-infections are responsible for the activation of the immune system [1], resulting in proinflammatory and pro-thrombotic status [2]. Pro-inflammatory high density lipoprotein $(\mathrm{HDL})$ is dysfunctional with high redox activity and easy non-calcified coronary atherosclerotic plaque rupture [3]. Endothelial and macrophage cell function is significantly impaired. Some antiretroviral agents (either directly or via associated dyslipidemia and insulin resistance) may also contribute to the increased rate of cardiovascular disease in HIV and therefore require careful selection according to the underlying cardiovascular risk factors.

A 35-year-old man was diagnosed (02.09.2009) with syphilis and HIV infection classified as stage A3. The patient was a cigarette smoker and had arterial hypertension and a family history of coronary artery disease (CAD). Combined antiretroviral therapy (CART) was composed of lamivudine, abacavir atazanavir and ritonavir. One year later atazanavir was replaced with darunavir due to potential drug-to-drug interaction with protein pump inhibitor (PPI). The patient remained asymptomatic and HIV RNA was undetectable. The CD4 T lymphocyte count was 386 cells/ $\mu$ l.

Due to exercise capacity impairment control transthoracic echocardiography (TTE) was performed and revealed: aneurysm of the ascending aorta with maximal diameter $60 \mathrm{~mm}$, aortic bulb $50 \mathrm{~mm}$, aortic annulus $26 \mathrm{~mm}$ with good left ventricular ejection fraction (LVEF) $-66 \%$. The aortic valve function was preserved. Preoperative coronarography revealed no significant atherosclerosis in coronary vessels. Initial lipid profile revealed hypertriglyceridemia (LDL 130 mg/dl, HDL 41 mg/dl, TG 240 mg/dl). Lipid-lowering therapy was initiated (atorvastatin $40 \mathrm{mg}$, fenofibrate $267 \mathrm{mg})$.
Supracoronary ascending aorta replacement with a vascular prosthesis (Vascutek 28) and aortic commissure suspension were performed (25.03.2011) without complications. The patient was discharged on the $5^{\text {th }}$ day after the surgery. The patient had outpatient check-ups regularly.

Eighteen months later (22.08.2012) the patient had lateral ST elevation myocardial infarction (STEMI). Coronary angiography revealed narrowing of the circumflex artery (CX), treated with bare metal stent (BMS) implantation. At that moment $50 \%$ de novo stenosis in the proximal segment of the left anterior descending artery (LAD) was also diagnosed (Fig. 1). Double antiplatelet therapy was introduced and continuation of antihypertensive and lipid-lowering medication was recommended. HIV RNA remained undetectable and the CD4 T lymphocyte count was 200300 cells/ $\mu$ l. Combined antiretroviral therapy was modified to tenofovir, emtricitabine, darunavir and ritonavir, due to the suspected adverse impact of abacavir on the cardiovascular risk.

Six months later (1.03.2013) the patient was admitted again to the cardiology department with diagnosis of NSTEMI. Coronary angiography revealed de novo 95\% narrowing in the $3^{\text {rd }}$ segment of the right coronary artery (RCA) (without progression in the proximal LAD), treated with BMS implantation. The lipid profile showed a good response to cholesterol-lowering treatment (LDL $49 \mathrm{mg} / \mathrm{dl}$, HDL $41 \mathrm{mg} / \mathrm{dl}$ ). Due to hypertriglyceridemia (TG $249 \mathrm{mg} / \mathrm{dl}$ ) lipid-lowering therapy was changed (rosuvastatin $40 \mathrm{mg}$ and fenofibrate $267 \mathrm{mg}$, BioCardine Omega-3). Lifestyle modifications (cigarette and alcohol cessation) were once again recommended to the patient. At that time the CD4 T lymphocyte count increased to 660 cells $/ \mu$ l.

In a 10-month follow-up the deterioration of angina pectoris (to CCS class III) was observed, and was confirmed with non-invasive tests. Coronary angiography revealed progression of proximal LAD stenosis (90\%), and elec-

Address for correspondence: Anna Marcinkiewicz, Department of Cardiac Surgery, Central Clinical Hospital, Medical University of Lodz, 251 Pomorska St, 92-013 Lodz, Poland, phone/fax: +48 4220144 60/61, e-mail: annamar87@o2.pl

Received: 16.02.2017, accepted: 4.06.2017. 


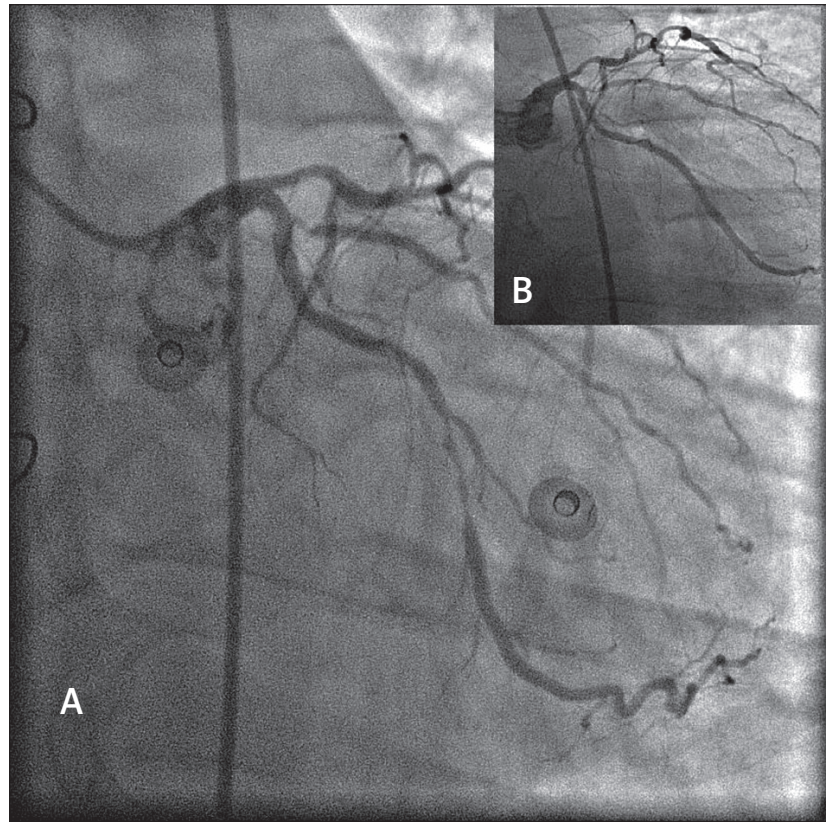

Fig. 1. Coronary angiography. Significant narrowing of $\mathrm{Cx}$, accompanied by $50 \%$ stenosis of LAD (A). Successful BMS implantation into $\mathrm{Cx}(\mathrm{B})$

$C x$ - circumflex artery, LAD - left anterior descending artery

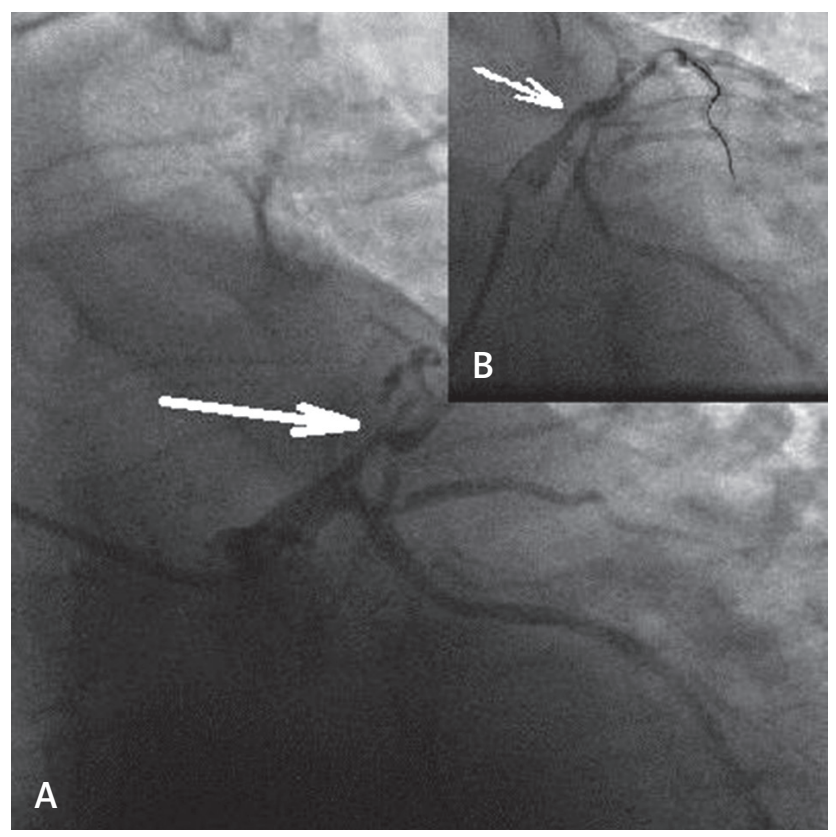

Fig. 2. Coronary angiography, LAO/CAU view. $90 \%$ proximal LAD lesion (A) and successful DES implantation (B) (white arrow)

DES - drug-eluting stent

tive stenting with a drug-eluting stent (DES) was applied (17.01.2014). Simultaneously a good long-term effect of the percutaneous angioplasty of the Cx and RCA was confirmed (Fig. 2). The lipid profile showed a good response to lipidlowering treatment (LDL $71 \mathrm{mg} / \mathrm{dl}$, HDL $44 \mathrm{mg} / \mathrm{dl}$, TG 149 $\mathrm{mg} / \mathrm{dl})$. Control echocardiography revealed good myocardi- al function (LVEF 55\%) without any valvular disorders. The patient was discharged on the second day in good condition on antiplatelet, antihypertensive and lipid-lowering medications.

It is estimated that in 2012, 35.3 million people were living with HIV worldwide (30.1 million adults - both sexes equally). It has been reported that a patient infected with HIV develops atherosclerosis noticeably earlier; therefore HIV infection is considered as an independent risk factor for cardiovascular disease. Underlying mechanisms are varied and not fully elucidated. A low level of CD4+ T lymphocytes is associated with decreased apolipoprotein B [4]. LDL-cholesterol is smaller than normal, harder to eliminate and prone to oxidation (proatherogenic small dense LDL) [5]. Hypertriglyceridemia is a result of HIV infection itself and interferon- $\alpha$ overproduction. Combined antiretroviral therapy accelerates dyslipidemia and insulin resistance, and this could contribute to the increased CAD risk. On the other hand, the atherogenic lipid profile depends on the antiretroviral drugs, especially protease inhibitors. However, such drugs as atazanavir and darunavir are associated with relatively low adverse lipid alterations and cardiac safety [6]. Recently introduced PCSK9 inhibitors, which are administered subcutaneously, could be a good alternative for patients with HIV on CART. This type of lipid-lowering drugs does not interfere with orally administered medications. Furthermore, nicotinic acid usage in HIV-infected patients could also have a positive additive value in improving the lipid profile and decreasing the risk of rapid development of atherosclerotic lesions [7].

The onset of CAD in HIV-infected individuals is usually earlier and its risk underestimated, so preventive practice should be introduced in patients under 45 years [8]. The analysis by Mehta and Khan [9] allowed them to establish some characteristic features of CAD in HIV-infected individuals: the median age of onset was 42.3 years, CAD was a late complication of HIV infection, the interval between HIV and CAD diagnosis was 72 months, no correlation with CD4 T lymphocyte count was found, acute myocardial infarction was the initial presentation in $77 \%$ of cases, and most patients had 3-vessel disease. We found many correlations mentioned above with our patient: CAD was diagnosed a little bit earlier, at the age of 38 years, but with myocardial infarction as the first manifestation, 3 years after HIV diagnosis. The relatively prompt clinical progression of atherosclerosis (significant stenosis of three major coronary arteries developed within 1.5 years, resulting in two myocardial infarctions) is probably due to the combination of many risk factors (HIV infection, smoking, arterial hypertension, dyslipidemia, family history of (AD), as well as the contribution of CART.

In HIV patients on CART who have a new cardiovascular event, antiretroviral therapy modification should be considered for improving the patient's prognosis. Treatment modifications would include replacement of protease inhibitor (PI) with non-nucleoside reverse transcriptase inhibitors (NNRTI) or with another PI with less metabolic disturbance 
- in our patient we replaced atazanavir and abacavir with darunavir and tenofovir.

The good efficacy of CART in HIV-infected patient survival with a cumulative impact of long-lasting HIV infection, adverse side effects of CART and traditional cardiovascular risk factors seem to contribute equally to the increasing prevalence of cardiovascular events. The onset of CAD seems to be earlier and the course more sudden than in the non-HIV population.

\section{Diclosure}

Authors report no conflict of interest.

\section{References}

1. Deeks SG Immune dysfunction, inflammation, and accelerated aging in patients on antiretroviral therapy. Top HIV Med 2009; 17: 118-123.

2. Ipp H, Zemlin AE, Erasmus RT, Glashoff RH. Role of inflammation in HIV-1 disease progression and prognosis. Crit Rev Clin Lab Sci 2014; 51: 98-111.
3. Zanni MV, Kelesidis T, Fitzgerald ML, Lo J, Abbara S, Wai B, Marmarelis E, Hernandez NJ, Yang OO, Currier JS, Grinspoon SK. HDL redox activity is increased in HIV-infected men in association with macrophage activation and noncalcified coronary atherosclerotic plaque. Antivir Ther 2014; 19: 805-811.

4. Riddler SA, Smit E, Cole SR, Li R, Chmiel JS, Dobs A, Palella F, Visscher B, Evans R, Kingsley LA. Impact of HIV infection and HAART on serum lipids in men. JAMA 2003; 289: 2978-29982.

5. Shahmanesh M, Das S, Stolinski M, Shojaee-Moradie F, Jackson NC, Jefferson W, Cramb R, Nightingale P, Umpleby AM. Antiretroviral treatment reduces very-low-density lipoprotein and intermediate-density lipoprotein apolipoprotein B fractional catabolic rate in human immunodeficiency virus-infected patients with mild dyslipidemia. J Clin Endocrinol Metab 2005; 90: 755-760.

6. Feeney ER, Mallon PWG. HIV and HAART-associated dyslipidemia. Open Cardiovasc Med J 2011; 5: 49-63.

7. Catapano AL, Graham J, De Backer G, Wiklund O, Chapman MJ, Drexel H, Hoes AW, Jennings CS, Landmesser U, Pedersen TR, Reiner Ž, Riccardi G, Taskinen MR, Tokgozoglu L, Verschuren WM, Vlachopoulos C, Wood DA, Zamorano JL; Authors/Task Force Members; Additional Contributor. 2016 ESC/EAS Guidelines for the Management of Dyslipidaemias. Eur Heart J 2016; 37: 2999-3058.

8. Boccara F, Lang S, Meuleman C, Ederhy S, Mary-Krause M, Costagliola D, Capeau J, Cohen A. HIV and coronary heart disease: time for a better understanding. Am Coll Cardiol 2013; 61: 511-523.

9. Mehta NJ, Khan IA. HIV-associated coronary artery disease. Angiology 2003; 54: $269-275$ 